\title{
PESQUISA-AÇÃO: UMA ALTERNATIVA METODOLÓGICA PARA PESQUISA EM ENFERMAGEM ${ }^{1}$ ACTION-RESEARCH: A METHODOLOGICAL OPTION FOR NURSING RESEARCH INVESTIGATIÓN-ACCIÓN: UNA ALTERNATIVA METODOLÓGICA PARA INVESTIGAR EN ENFERMERÍA
}

\author{
Luciana Grittem², Marineli Joaquim Meier', Ivete Palmira Sanson Zagonel ${ }^{4}$
}

\footnotetext{
${ }^{1}$ Trabalho resultante da disciplina Concepções Teórico-Filosóficas e Metodológicas para a Prática Profissional em Enfermagem do Curso de Mestrado em Enfermagem da Universidade Federal do Paraná (UFPR).

${ }^{2}$ Mestranda do Programa de Pós-Graduação da UFPR. Enfermeira do Hospital de Clínicas da UFPR. Paraná, Brasil.

${ }^{3}$ Doutora em Filosofia de Enfermagem. Professora do Curso de Graduação em Enfermagem e Programa de Pós-Graduação da UFPR. Paraná, Brasil.

${ }^{4}$ Doutora em Filosofia de Enfermagem. Docente do Programa de Pós-Graduação em Saúde da Criança e do Adolescente da Faculdade Pequeno Príncipe. Paraná, Brasil.
}

PALAVRAS-CHAVE: Enfermagem. Pesquisa. Metodologia.
RESUMO: Trata-se de uma reflexão sobre pesquisa-ação, realizada a partir de intensa revisão da literatura e banco de dissertações e teses as quais apontam para uma nova possibilidade metodológica de pesquisa na enfermagem. Existem divergências sobre a aplicabilidade da maioria das pesquisas na prática, devido ao distanciamento do pesquisador da realidade estudada, entretanto na pesquisaação há a inserção do pesquisador no campo de coleta de dados. A pesquisa-ação pode e deve ser utilizada na área de saúde, especialmente pela enfermagem, em questões em que há interesse coletivo para a resolução de problemas, para mobilizar os profissionais para uma prática crítica e reflexiva. Entre as limitações deste tipo de pesquisa, pode-se citar o tempo prolongado para obtenção de resultados, a manutenção da imparcialidade do pesquisador no desenvolvimento de todas as etapas da pesquisa. A utilização de referenciais metodológicos apropriados à prática de enfermagem é de suma importância para o desenvolvimento da área.
KEYWORDS: Nursing. Research. Methodology.

\begin{abstract}
This study is a reflection upon action-research, carried out through exhaustive literature, graduate thesis, and dissertation review, which point to a new methodological possibility in nursing research. There are some divergences about the applicability of the majority of these studies in practice, due to the researcher's detachment from the studied reality. However, in action-research, there is his/ her insertion into the data collection field. Action-research can and must be used in health care, especially nursing, in questions in which there is collective interest for problem solving in order to mobilize professionals towards a critical and reflective practice. Among the shortcomings of this kind of research, the prolonged time in obtaining results and maintaining researcher impartiality in developing all the research steps can be cited. The utilization of adequate methodological references for nursing practice is extremely important for the development of this area.
\end{abstract}

PALABRAS CLAVE: Enfermería. Pesquisa. Metodología.
RESUMEN: Se trata de una reflexión sobre investigación-acción, realizada a partir de una intensa revisión de la literatura y del banco de tesinas y tesis, las cuales señalan una nueva posibilidad metodológica de investigación en enfermería. Existen divergencias sobre la aplicabilidad de la mayoría de las investigaciones en la práctica, debido al distanciamiento del investigador de la realidad estudiada, sin embargo, en la investigación-acción se produce la participación del investigador en el campo de recolección de los datos. La investigación-acción puede y debe ser utilizada en el área de la salud, especialmente por la enfermería, en cuestiones en las que hay interés colectivo para la resolución de problemas, para movilizar los profesionales para una práctica crítica y reflexiva. Entre las limitaciones de este tipo de investigación, se pueden citar: el prolongado tiempo para la obtención de los resultados, el mantenimiento de la imparcialidad del investigador en el desarrollo de todas las etapas de la investigación. La utilización de referencias metodológicas apropiadas para la práctica de enfermería es sumamente importante para el desarrollo del área.
Luciana Grittem

Endereço: Alberto Rutz, 506

81320-280 - Curitiba, PR, Brasil

E-mail: lug_hc@yahoo.com.br
Reflexão

Recebido em: 12 de dezembro de 2007 Aprovação final: 13 de outubro de 2008 


\section{INTRODUÇÃO}

Para o desenvolvimento de pesquisa na prática profissional de enfermagem, é importante ter consciência dos referenciais metodológicos, da definição clara e objetiva do objeto de estudo, para a seleção da metodologia mais adequada a ser aplicada. Para se obter respostas e resultados aos seus questionamentos, a enfermagem busca ampliar a produção de conhecimentos, por meio da realização de pesquisas. Entre estas estratégias metodológicas está a pesquisa-ação, que é utilizada quando há interesse coletivo na resolução de problemas. Assim esse artigo, pretende refletir sobre a pesquisa-ação como uma possibilidade metodológica de pesquisa na enfermagem.

A origem deste tipo de pesquisa ainda é incerta, no entanto atribui-se o início de todo o processo às dinâmicas de grupo de Kurt Lewin, oriundo da psicologia social, nos anos 40, com a formação dos T-groups (grupos de treinamento), nos quais eram realizadas reflexões sobre suas ações, como meio de desenvolver o conhecimento para melhor atuação do indivíduo. ${ }^{1}$ A pesquisaação teve influências das linhas positivistas nos anos de 1946 e 1948, em que desmembra os enfoques em diagnóstica, participante, empírico e experimental, assim como descaracteriza suas raízes epistemológicas. A partir desta fragmentação, surgiram várias pesquisas com as mesmas características, aspecto que levou ao enfraquecimento e até mesmo desconhecimento de sua origem, por parte de muitos pesquisadores. $^{2}$

A pesquisa-ação tem sido utilizada no desenvolvimento de pesquisas em diversas áreas como na educação, política, agronomia, publicidade e propaganda, assim como na área organizacional, bancária e de saúde. ${ }^{2-5} \mathrm{Na}$ área da saúde, esse tipo de pesquisa é utilizado em especial nos programas de saúde coletiva. Devido à significativa participação popular, torna-se um instrumento valioso, pois ocorre uma construção social de conhecimento, por meio da interação e cooperação dos atores. ${ }^{6}$

A dificuldade de definir a pesquisa-ação ocorre devido ao fato de que ela se apresenta tão natural no seu desenvolvimento e ao mesmo tempo assume aspectos diferentes, para distintas aplicações. ${ }^{7}$ Ela é considerada um modelo de pesquisa associada a diversas formas de ações coletivas, orientadas para a resolução de problemas ou com objetivo de transformação. Os adeptos da metodologia convencional receiam o rebaixamento do nível de exigência com a pesquisa-ação e a pesquisa participante, porém tais riscos também ocorrem em outros tipos de pesquisa e podem ser superados mediante um embasamento metodológico adequado. ${ }^{8}$

Existem confusões acerca da pesquisa-ação e pesquisa-participante devido ao fato de ambas terem a mesma origem, apesar da discordância de alguns autores, mas existem muitas diferenças entre esses dois tipos de pesquisa quando são verificadas as etapas dos métodos. Na pesquisa-ação, além da participação dos sujeitos, se propõe uma forma de ação planejada, de caráter diversificado conforme a área de aplicação, o que nem sempre acontece na pesquisa-participante. ${ }^{8}$

As pesquisas participativas constituem um distanciamento do modelo tradicional de pesquisa acadêmica, pois o problema estudado não tem origem em teorias ou na literatura, como ocorre na pesquisa convencional. Nas pesquisas em que se prevê a participação dos sujeitos busca-se na prática, a problemática a ser discutida, como é o exemplo da pesquisa-ação, porém não são bem vistas por alguns pesquisadores sociais e de outras áreas. Desta forma, ainda prevalecem as técnicas convencionais de pesquisa.

Muitos pesquisadores temem estes tipos de metodologias, pois as consideram menos científicas e mais expostas à manipulação, porém isto fica sob o controle do método e da ética utilizados pelo pesquisador. As pesquisas que possuem caráter participativo e de capacitação bem conduzidos têm efeitos significativos nas trajetórias de vida dos grupos envolvidos na pesquisa. ${ }^{6}$

A enfermagem tem ampliado a produção de conhecimentos por meio da realização de pesquisas. Dentre as estratégias adotadas, destaca-se a pesquisa-ação, visto a sua aplicabilidade em questões em que há interesse coletivo para a resolução de problemas. Sua utilização tem sido ampla na enfermagem, nas áreas da saúde coletiva, hospitalar, obstetrícia, psiquiatria e docência, entre outras. Seu desenvolvimento prevê a resolução de situações cotidianas e que requerem mudança. Desafia e estimula os pesquisadores enfermeiros a compreenderem seus conceitos, se aventura por outros caminhos metodológicos..$^{9-14}$

A participação dos membros da equipe de enfermagem em fóruns de discussão é bastante comum; e as ações desta categoria profissional somente têm consistência quando são construídas coletivamente, de outro modo não se obtém efetividade. Portanto, considera-se a metodologia de pesquisa-ação como uma abordagem original e apropriada a ser aplicada à enfermagem, pois 
visa mudança de rotinas, implantação de serviços, modificação de comportamento de um grupo de indivíduos, em que os sujeitos são co-responsáveis pela ação realizada.

Neste sentido será abordado neste artigo, o histórico, definições, aplicabilidade em diversas áreas, etapas para o planejamento da pesquisaação e possíveis contribuições que este tipo de pesquisa oferece à enfermagem.

\section{HISTÓRICO, DEFINIÇÕES E ETAPAS DA PESQUISA-AÇÃO}

Na observação das etapas do planejamento da pesquisa-ação, é possível visualizá-la nas pesquisas de enfermagem, tendo em vista que são desenvolvidas em sua maioria na informalidade do cotidiano das atividades. Cabe ao enfermeiropesquisador cumprir os requisitos do método, para obter reconhecimento de seu trabalho no meio acadêmico, mostrar que é possível reunir prática e teoria, numa relação de crescimento e reconhecimento.

Ao optar por este tipo de pesquisa, é necessário ter em mente o tempo que o pesquisador terá para desenvolvê-la, pois é imprescindível o cumprimento de todas as fases, não sendo possível realizar somente a fase de diagnósticos, pois o que caracteriza a pesquisa-ação é a mudança. A pesquisa pode custar muito tempo, quando se pretende que os sujeitos da pesquisa realizem o levantamento de seus problemas e necessidades, pois diferente do refletir, o fazer torna-se mais difícil e emocionalmente cansativo para os sujeitos. ${ }^{5}$

O planejamento na pesquisa-ação é flexível e não segue uma série de fases ordenadas, de forma rígida. Assim, existem diversas etapas para organização e efetivação e sua execução pode ocorrer de diversas formas.

As fases desse tipo de pesquisa estão relacionadas abaixo, de maneira resumida, pois para entendimento deste método é necessário um estudo mais minucioso: ${ }^{8}$

- Fase exploratória: diagnóstico da realidade do campo de pesquisa, levantamento da situação e dos problemas. Pesquisadores e participantes estabelecem os objetivos da pesquisa, interligando os problemas, campo de observação, atores e tipo de ação que se pretende focalizar;

- Tema da pesquisa: deve interessar ao pesquisador e aos sujeitos investigados, para que todos desempenhem um papel eficiente no desenvolvimento da pesquisa. $\mathrm{O}$ tema pode ser solicitado pe- los atores da situação. Neste momento é escolhido um marco teórico para nortear a pesquisa.

- Colocação dos problemas: nessa fase há discussão sobre a relevância científica e a prática do que será pesquisado.

- O lugar da teoria: articulação com um referencial teórico de acordo com o local onde será realizada a pesquisa. As informações que serão levadas ao seminário (explicitado baixo), estratégia que faz parte do método, devem ser interpretadas conforme esta teoria, dando rigor científico à pesquisa.

- Hipóteses: são suposições formuladas pelo pesquisador a respeito de possíveis soluções para um problema colocado na pesquisa, assumindo caráter de condução do pensamento.

- Seminário: tem a finalidade de promover discussão e tomada de decisões acerca da investigação (definição de temas e problemas), constituir grupos de estudos, definir ações, acompanhar e avaliar resultados. O seminário tem a função de coordenar as atividades do grupo, sempre finalizado pela confecção de atas das reuniões.

- Campo de observação, amostragem e representatividade qualitativa: pode abranger uma comunidade geograficamente concentrada ou dispersa. A amostragem e representatividade qualitativa são discutíveis.

- Coleta de dados: as principais técnicas utilizadas para a coleta de dados são a entrevistas coletiva ou individual, questionários convencionais, estudos de jornais e revistas. Podem ser montados diversos grupos de observação e para isso faz-se necessário treinamento deste grupo. Todas as informações coletadas são transferidas ao seminário, para discussão, análise e interpretação.

- Aprendizagem: as ações investigadas envolvem produção e circulação de informações, tomada de decisões, supondo uma capacidade de aprendizagem dos participantes. Esta capacidade é aproveitada e enriquecida, já que ocorre uma aprendizagem conjunta.

- Saber formal e saber informal: deve melhorar a comunicação entre os universos culturais, dos especialistas e o dos interessados. Há uma interação entre o saber prático e o teórico, que se funde na construção de novos conhecimentos. Busca-se a intercompreensão.

- Plano de ação: visa definir os atores, a relação entre eles, quem são os líderes, quais os objetivos e os critérios de avaliação da pesquisa, continuidade frente às dificuldades, quais estratégias 
serão utilizadas para assegurar a participação dos sujeitos, incorporação de sugestões e qual a metodologia de avaliação conjunta de resultados.

- Divulgação externa: nessa fase ocorre o retorno dos resultados da pesquisa aos participantes, divulgação dos resultados em eventos, congressos, conferências e publicações científicas.

A pesquisa-ação compõe-se de doze fases descritas anteriormente, que se sobrepõem e integram-se de forma muito maleável. Estas fases devem ser vistas como ponto de partida e chegada, sendo possível em cada situação, o pesquisador junto com os participantes, redefinir e adaptar de acordo com as circunstâncias da situação investigada. ${ }^{8}$ Esse aspecto precisa ser considerado e utilizado no desenvolvimento da pesquisa, isto porque, a sobreposição e interligação das fases atribuem dinamismo a todo o processo.

Uma limitação no desenvolvimento desta metodologia, talvez seja o tempo necessário para a efetivação das ações propostas. Ela não é recomendada em estudos, no qual haja um período razoavelmente restrito para atender às demandas suscitadas pela questão de pesquisa. Outra dificuldade encontrada é a manutenção da imparcialidade do pesquisador, frente às discussões do grupo, no estímulo da construção coletiva e na apuração dos resultados. A análise dos dados deve ser ética e não tendenciosa ao alcance dos objetivos desejados na pesquisa, tendo em vista que os resultados emergentes do grupo eventualmente divergem do proposto pelo pesquisador.

Na interação pesquisador-pesquisado, ocorre a integração dos diferentes saberes e posições que eles possuem, sem diferenciação hierárquica. No quadro profissional, a pesquisa é conduzida de modo que não seja predeterminado pelos interesses dominantes da organização, mas como um constante compromisso com a verdade e com a intercompreensão dos atores que se relacionam na situação investigada em que o pesquisador não é tendencioso. ${ }^{8}$

\section{CONTRIBUIÇÕES PARA A ENFERMA- GEM}

A pesquisa-ação pode e deve ser utilizada na área de saúde, especialmente pela enfermagem, na discussão sobre a prática profissional, para mobilizar os profissionais para uma prática crítica e reflexiva. Comparada aos estudos acadêmicos, planejados e executados por outros profissionais, esta metodologia tem sido mais fluida e menos rigorosa, o que pode conduzir as pessoas que não estão envolvidas com a pesquisa, a considerá-la abstrata. Contudo, é considerada aproximadamente sete vezes mais difícil do que a pesquisa convencional. ${ }^{5}$

Toda pesquisa-ação é considerada participativa sendo necessário o envolvimento das pessoas implicadas nos problemas e, somente é considerada como tal, se houver uma ação por parte do grupo de pessoas sob observação. Essa ação por sua vez, deverá ter um caráter prático e não apenas prosaico. ${ }^{8}$

O descritor da pesquisa-ação não está cadastrado na Biblioteca Virtual de Saúde, o que dificulta a localização de estudos que utilizaram esta metodologia, porém encontrou-se em algumas pesquisas de enfermagem, em bancos de dissertações e teses e em revistas de enfermagem indexadas na Scientific Eletronic Library Online (SciELO), a utilização desta metodologia. Sua aplicabilidade destaca-se principalmente nas áreas da educação e psicologia. Estas áreas encontram neste referencial subsídio às necessidades de emancipação, pessoal, política e social. ${ }^{2,5,7}$

Na enfermagem existem divergências sobre a aplicabilidade da maioria das pesquisas na prática, devido ao distanciamento do pesquisador da realidade estudada, entretanto na pesquisa-ação há a inserção do pesquisador no campo de coleta de dados. Este método de pesquisa vem mostrar a possibilidade, da utilização do conhecimento dos participantes, ou seja, dos sujeitos envolvidos na pesquisa, como agente colaborador e capaz de propor e implementar mudanças de suas atividades práticas, o que gera satisfação, possivelmente superior às pesquisas convencionais que apresentam relatórios e dados, sem o comprometimento com resultados.

A pesquisa-ação tende a ser pragmática, se distinguindo da pesquisa científica tradicional, mas também é limitada pelo contexto da ética e da prática. ${ }^{7}$ Vale ressaltar que, em toda e qualquer proposta de mudança, há necessidade de que esta não seja pensada somente pelo pesquisador ou pelo superior hierárquico, pois desta forma será negada a dimensão dialética e crítica da pesquisa. Ela deve gerar reflexão coletiva, considerar a voz dos sujeitos, não apenas para interpretação posterior, mas como metodologia de investigação.

As críticas de grupos profissionais autoconscientes de sua prática é de fundamental importância, para que todos os participantes aprendam em contato com os outros, aceitem 
e relativizem seus pontos de vista. Capacitam as pessoas na realização de tarefas, fortalecem planos e atividades para que os indivíduos sejam capazes de realizá-los sozinhos. ${ }^{6}$

Um dos pressupostos da pesquisa-ação é que os indivíduos envolvidos nela compõem um grupo com objetivos e metas em comum. Percebe-se que na ação, o agir está vinculado ao modo de ser e é possível identificar o que se é no que se faz, pois as ações são práticas sociais e constituem-se a partir da história de cada sujeito. ${ }^{4}$ Com esta metodologia é possível testar teorias do comportamento humano no mundo real, dar feedback aos pesquisadores sobre a utilidade de seus trabalhos, e ensinar pesquisadores como conduzir pesquisas de campo. ${ }^{8}$

A pesquisa-ação se mostra como uma possibilidade metodológica na enfermagem, pois envolve pessoas na resolução de problemas, desenvolve grupos interessados em mudanças, reduz a distância entre o pesquisador e os sujeitos da pesquisa e assegura que a pesquisa não se torne assunto para poucas pessoas. Por embasar as mudanças em fatos e não apenas em ideologias, tem caráter emancipatório, que ocorre por meio do reconhecimento profissional, com a superação de obstáculos.

A aplicabilidade da referida metodologia em estudos na enfermagem, quando se deseja uma aproximação da teoria à prática, é viável. Um exemplo disto é a sua utilização em trabalhos nos quais se pretende a construção coletiva de mudanças, instrumentos, conceitos e comportamentos. É possível destacar sua aplicação nos estudos em que se utilizou esta metodologia para o desenvolvimento e implantação da Sistematização da Assistência de Enfermagem em instituições hospitalares. ${ }^{10,15}$ Além destas, as áreas em que se prevê o desenvolvimento de educação popular ou de uma determinada comunidade, a metodologia em questão, mostra-se como uma estratégia que atende as necessidades dos sujeitos e do pesquisador no alcance dos objetivos planejados nas pesquisas.

\section{REFERÊNCIAS}

1. Origem da pesquisa-ação - dinâmica de grupos. [acesso em 2007 Nov 27]. Disponível em: http:/ / pt.wikipedia.org/wiki/Din\%C3\%A2mica_de_ grupos.

2. Franco MAS. Pedagogia da pesquisa-ação. Educação e Pesquisa [periódico na Internet]. 2005 Set-Dez [acesso em 2008 Out 06]; 3(3):483-502. Disponível em: http:/ / www.scielo.br/scielo.php?pid=S151797022005000300011\&script=sci_arttext.

3. Parker I. A desconstrução da psicopatologia na pesquisa ação. Mental [periódico na Internet]. 2004 Nov [acesso em 2007 Nov 28]; 3(II):15-29. Disponível em: http://pepsic.bvs-psi.org.br/pdf/mental/ v2n3/v2n3a02.pdf.

4. Pimenta SG. Pesquisa-ação crítico colaborativa: construindo seu significado a partir de experiências com a formação de docentes. Educação e Pesquisa [periódico na Internet]. 2005 Set-Dez [acesso em 2007 Nov 28]; 3(3):521-39. Disponível em: http:/ / www.scielo.br/pdf/ep/v31n3/a13v31n3.pdf.

5. Sommer R, Amick T. Pesquisa-ação: ligando pesquisa à mudança organizacional. [Série: Planejamento de Pesquisa nas Ciências Sociais. n.4]. Brasília (DF):UnB; 1984. [acesso em 2006 Jul 17]. Disponível em: http://www.unb.br/ip/lpa/ pdf/04Action.pdf.

6. Thiollent M. Construção do conhecimento e metodologia da extensão. In: Anais do I Congresso Brasileiro de Extensão Universitária, 2002 Nov 10; João Pessoa, PB. João Pessoa: UDUFPB; 2003 [acesso em 2006 Jun 03]. Mesa redonda. Disponível em: http://rtprac.prac.ufpb.br/anais/anais/ficha.html.

7. Tripp D. Pesquisa-ação: uma introdução metodológica. Educação e Pesquisa [periódico na Internet]. 2005 Set-Dez [acesso em 2007 Nov 28]; 31(3):443-6. Disponível em http:/ / www.scielo.br/ pdf/ep/v31n3/a09v31n3.pdf.

8. Thiollent M. Metodologia da pesquisa-ação. $14^{\mathrm{a}} \mathrm{ed}$. São Paulo (SP): Cortez, 2005.

9. Grittem L. Sistematização da assistência perioperatória: uma tecnologia de enfermagem [dissertação]. Curitiba (PR): Universidade Federal do Paraná. Setor de Ciência de Saúde, 2007.

10. Gonçalves, VLM, Leite MMJ, Ciampone, MHT. A pesquisa-ação como método para reconstrução de um processo de avaliação de desempenho. Cogitare. 2004 Jun; 9(1):50-9.

11. Andrade JS de, Vieira MJ. Prática assistencial de enfermagem: problemas, perspectivas e necessidades de sistematização. Rev Bras Enferm. 2005 Mai-Jun; 58(3):261-5.

12. Reberte LM, Hoga LAK. O desenvolvimento de um grupo de gestantes com utilização da abordagem corporal. Texto Contexto Enferm [periódico na Internet]. 2005 Abr-Jun [acesso em 2008 Out 06]; 14(2):186-92. Disponível em: http://www. scielo.br/scielo.php?script=sci_pdf\&pid=S010407072005000200005\&lng=em \&nrm=1\&tlng=pt.

13. Hoga, LAK, Reberte LM. Técnicas corporais em Grupo de Gestantes: a experiência dos participantes. Rev Bras Enferm [periódico na Internet]. 2006 Maio-Jun [acesso em 2007 Nov 30]; 59(3):308-13. Disponível em: http:/ / www.portalbvsenf.eerp.usp. br/ pdf/reben/v59n3/a11.pdf. 
14. Frota MA, Albuquerque, C de M, Linard AG. Educação popular em saúde no cuidado à criança desnutrida. Texto ContextoEnferm [periódicona Internet].2007 AbrJun [acesso em 2007 Nov 30]; 16(2): 246-53. Disponível em: http://www.scielo.br/scielo.php?script=sci arttext\&pid=S0104-07072007000200006\&lng $=$. doi:10.1590/S0104-07072007000200006.

15. Crossetti MG, Portella V, Saurin G, Thompson DB, Alves TSR, Marini M. O uso da informática nas práticas de cuidado: construindo modelos de registro com foco nos diagnósticos de enfermagem em centro cirúrgico do Hospital de Clínicas de Porto Alegre. In: Anais da $24^{a}$ Semana Científica do HCPA, 2004 Set 13-17; Porto Alegre. Revista HCPA [online]. Porto Alegre 2004 [acesso em 2007 Nov 30]; 24 (suplemento): 122. Disponível em: http://www.hcpa.ufrgs.br/downloads / RevistaCientífica/2004/ANAIS_2004.pdf. 